\title{
2012: das Ende der Welt? Von wegen!
}

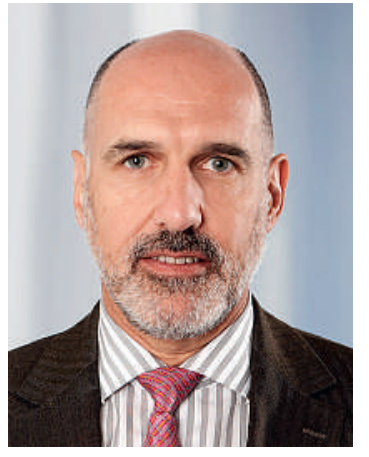

Nein, 2012 wird die Welt nicht untergehen. Doch langweilig dürfte es Ihnen auch nicht werden: Falls Sie Abenteuer mögen, wird 2012 Ihr Jahr sein!

Das politische Programm im Gesundheitsbereich, das im neuen Jahr ansteht, ist in der Tat bemerkenswert und wird uns mehr als genug beschäftigen. Denn der Sinn und Zweck der FMH ist es, der Ärzteschaft in der Schweiz die bestmöglichen Arbeitsbedingungen und eine optimale Lebensqualität zu sichern. Und dabei auf die Vielfalt der Meinungen und Lebenslagen ihrer Mitglieder Rücksicht zu nehmen.

Erst kürzlich habe ich darauf hingewiesen, dass wir als Ärztinnen und Ärzte eine Reihe von gemeinsamen Nennern haben, vor denen die Unterschiede verblassen. Erwähnt hatte ich in diesem Zusammenhang die zentrale Stellung der Patientinnen und Patienten in der Medizin, den Vorrang der Arzt-Patienten-Beziehung vor allen anderen Alltagsaufgaben unseres Berufs, den Qualitätsanspruch - diese Grundsätze unserer Politik werden wir 2012 selbstverständlich beibehalten. In welchen Bereichen wird sie zur Anwendung gelangen?

\section{Wir als Ärztinnen und Ärzte haben eine Reihe von gemeinsamen Nennern, vor denen die Unterschiede verblassen.}

Zweifellos bei der Vorlage zur integrierten Versorgung: Wir stehen vor einer mehrmonatigen Abstimmungskampagne, die wir unbedingt gewinnen müssen und auf die wir uns bereits sehr aktiv vorbereiten. wurde das Referendum angekündigt. Auch mit diesem grundlegenden Thema wird sich die FMH beschäftigen.

Zudem müssen wir die Einführung der neuen Spitalfinanzierung und der SwissDRG-Fallpauschalen sehr aufmerksam verfolgen. Entsprechende Studien laufen bereits, um die Auswirkungen im Auge zu behalten. Wie Sie wissen, steht nächstes Jahr überdies die TARMED-Revision auf dem Programm. Für dieses wichtige Geschäft unseres Tarifdienstes, bei dem einiges auf dem Spiel steht, lautet das Motto Fortschritt und Rücksicht.

\section{Für die Vorlage zur integrierten Ver- sorgung stehen wir vor einer mehr- monatigen Abstimmungskampagne, die wir unbedingt gewinnen müssen.}

Ohne Anspruch auf Vollständigkeit zu erheben, muss ich mindestens zwei weitere Themen noch erwähnen:

Das eine ist die Initiative für eine öffentliche Krankenkasse, die nächstens eingereicht werden soll und zu der die Ärzteschaft wird Stellung beziehen müssen. Zunächst muss aber abgeklärt werden, ob unsere Delegiertenversammlung oder die Ärztekammer diesen Beschluss fassen kann oder ob erneut eine Urabstimmung durchgeführt werden sollte - was mir wünschenswert erscheint -, bevor diese Initiative vor das Volk kommt.

Das andere ist die immer drängendere Frage der Ärztedemografie. Die Aufhebung des Zulassungsstopps einerseits und der immer offensichtlichere Mangel an Schweizer Ärztinnen und Ärzten andererseits erfordern dringend konstruktive und realistische Lösungen. Im Rahmen einer Arbeitsgruppe, die gegenwärtig zusammengestellt wird, werden wir versuchen, entsprechende Vorschläge auszuarbeiten.

Und damit habe ich den Amtsantritt des neuen Gesundheitsministers und die Wiederwahl des Zentralvorstands noch gar nicht erwähnt, die ebenfalls Farbe in das neue Jahr bringen werden!

\section{Wenn sich der Wert eines kommenden Jahres an seinem Potential misst, an dem, was es mit sich bringen kann, dann verspricht 2012 jetzt schon einiges!}

Doch die Liste der Geschäfte, für die wir uns engagieren werden, ist um einiges länger!

$\mathrm{Zu}$ nennen ist hier die Initiative zur Hausarztmedizin, mit der sich das Parlament in einigen Wochen befasst, bevor sie dem Volk vorgelegt wird. Auch mehrere wichtige Geschäfte wie das Präventionsgesetz, das Gesetz über das elektronische Patientendossier und Invalidenversicherungsgesetz werden von uns entschlossene Interventionen erfordern. Und natürlich steht die KVG-Revision an, mit der die Weitergabe der Diagnosen an die Versicherer vorgeschrieben werden soll. Gegen diese Revision
Wenn sich der Wert eines kommenden Jahres an seinem Potential misst, an dem, was es mit sich bringen kann, dann verspricht 2012 jetzt schon einiges!

Nicht, dass für 2012 schon etwas feststehen würde, ganz im Gegenteil: Alles ist noch völlig offen.

Doch vielleicht sollte genau daran letztlich gemessen werden, was ein neues Jahr bringen kann. Wir werden uns jedenfalls an die Arbeit machen!

Mit den besten Wünschen des Zentralvorstands Dr. med. Jacques de Haller, Präsident der FMH 